\title{
Antimicrobial Compounds of Plant Origin as Efflux Pump Inhibitors: New Avenues for Controlling Multidrug Resistant Pathogens
}

\author{
Rao $\mathbf{M}^{1}$, Padyana $\mathbf{S}^{1}$, Dipin $\mathrm{KM}^{2}$, Kumar $\mathbf{S}^{2^{*}}$, Nayak BB ${ }^{2}$ and Varela $\mathbf{M F}^{3}$ \\ ${ }^{1}$ Department of Post-Graduate Studies in Dravyaguna Vijnana, Alva's Ayurveda Medical College, Karnataka, India \\ ${ }^{2}$ Post-Harvest Technology Department, QC Laboratory, ICAR-Central Institute of Fisheries Education (CIFE), Mumbai, India \\ ${ }^{3}$ Department of Biology, Eastern New Mexico University, Portales, USA
}

"Corresponding author: Sanath Kumar, Senior Scientist, QC Laboratory, Post-Harvest Technology Department, ICAR-Central Institute of Fisheries Education (CIFE), Versova-400061, Mumbai, India, Tel: +91-22-26361446; E-mail: sanathkumar@cife.edu.in

Received date: January 1, 2018; Accepted date: January 25, 2018; Published date: January 27, 2018

Copyright: @ 2018 Rao M, et al. This is an open-access article distributed under the terms of the Creative Commons Attribution License, which permits unrestricted use, distribution, and reproduction in any medium, provided the original author and source are credited.

\begin{abstract}
The emergence of multiple drug resistant pathogenic bacteria has severely constricted the antimicrobial options of treating infectious diseases. One of the powerful mechanisms of antibacterial resistance employed by the antibiotic resistant bacteria is the active extrusion of antimicrobials with the help of membrane transporters known as efflux pumps. Efflux pumps effectively reduce the intracellular concentrations of antimicrobials by their antiporter activity in which antimicrobials are extruded outside the bacterial cell using energy derived from ionic gradient across the cell membrane. While a few efflux pumps are capable of conferring clinical levels of resistance to antibiotics, while many others only marginally increase the minimum inhibitory concentrations (MIC) of antibiotics. However, the role of efflux pumps in gradual development of antibiotic resistance by pathogenic bacteria due to mutations and other molecular mechanisms during the course of antimicrobial therapy is well recognized. The inhibition of active efflux can result in maintenance of an intracellular concentration of antibiotics necessary to inhibit or kill bacteria. Plant-derived compounds have historically been recognized as effective antimicrobial agents. Advances in analytical techniques have enabled purification of natural compounds responsible for efflux pump inhibition and these compounds and their derivatives can act as lead compounds for designing more effective efflux pump inhibitors. Efflux pump inhibition is promising as an effective method of confronting bacterial resistance to antimicrobials either alone or as adjuvants with antibiotics, and thereby restoring the antibacterial efficacy of antibiotics.
\end{abstract}

Keywords: Efflux pump inhibition; Antibiotic resistance; Plant compounds; Antibiotic potentiation; Antiporter; Secondary active transporter

\section{Introduction}

For over seven decades after the introduction of the first antibiotic penicillin for treatment of infectious diseases, antibiotics have remained as sole weapons against pathogenic bacteria. However, the power of antibiotics is fast waning due to the rapid development of resistance by the pathogenic bacteria [1]. Apart from their use in the treatment of human infections, antibiotic have been used extensively in food animals, agriculture and aquaculture. A large proportion $(>70 \%)$ of globally produced antibiotics are used in animals for purposes of growth promotion, prophylaxis, improvement of feed efficiency and the treatment of infections [2]. The antibiotic overuse has gradually led to the development of resistance among human pathogenic bacteria. methicillin resistant Staphylococcus aureus (MRSA), vancomycin resistant Enterococcus faecalis (VRE), extremely drug resistant Mycobacterium tuberculosis, carbapenem resistant Enterobacteriaceae (CRE), New Delhi metallo- $\beta$-lactamase (NDM)-producing Klebsiella pneumoniae have all challenged the efficacy of antimicrobial chemotherapy [3-5]. Considerable research efforts are being focused on the development of new and effective chemotherapeutic agents. However, in the context of fast evolving antibiotic resistance mechanisms, it is critical to preserve the efficacy of available antibiotics by prudent and judicious use.

\section{Overview of bacterial resistance mechanisms}

Bacteria have evolved diverse mechanisms to overcome antibiotic pressure. Although exposure to antibiotics is a key reason for the development of antibiotic resistance, bacteria may also be intrinsically resistant to certain antimicrobials [6]. The common mechanisms of bacterial resistance to antibiotics include i) Enzymatic degradation of the antibiotics with the help of enzymes which hydrolyze antibiotics into ineffective compounds, (ii) Enzymatic modification of cellular targets of antibiotics such as the ribosomal protein subunits, nucleic acids, metabolic enzymes and the bacterial cell wall components so that the antibiotic is unable to effectively bind to its intended target, (iii) modification of cell membrane leading to reduced permeability of the antibiotic into the cytoplasm of the bacterial cell, (iv) active extrusion of the antibiotics by membrane efflux pumps thereby reducing the cellular concentrations of antibiotics to levels insufficient to kill or halt the growth of bacteria [6,7]. Bacteria may be single drug or multidrug resistant (MDR). MDR bacteria possess multiple mechanisms of antibiotic resistance and such abilities are encoded on mobile genetic elements such as the transposons and plasmids [8]. Horizontal transmission of the genetic elements such as plasmid and transposons through molecular mechanisms such as transformation, conjugation or phage-mediated transduction are responsible for the acquisition of multiple mechanisms of drug resistance [7,9]. On the other hand, many bacteria are intrinsically resistant to certain antibiotics. A classic example of intrinsic resistance is the antimicrobial resistance conferred by the chromosomally encoded marRAB locus of Escherichia coli [10]. This genetic element is responsible for the 
intrinsic resistance of Escherichia coli to diverse antibiotics including penicillins, cephalosporins, fluorquinolones, tetracyclines, chloramphenicol, nalidixic acid, rifampin etc. [11]. The AcrAB efflux pump located on the mar locus is responsible for the antibiotic resistance phenotype of the Gram-negative bacteria harboring marRAB locus $[11,12]$.

\section{Secondary active transporters}

Bacterial mechanism of transporting structurally diverse molecules across the membrane involves versatile membrane transporters classified as primary and secondary active transporters. The primary active transporter proteins use the energy derived from the hydrolysis of ATP, while the secondary active transporters make use of an ionic gradient across the membrane to energize the transport of solutes against a concentration gradient [13]. Secondary active transporters utilize the energy gradient created by $\mathrm{H}^{+}$or $\mathrm{Na}^{+}$across the membrane and transport diverse molecules such as salts, sugars, vitamins, fatty acids, amino acids, Kreb's cycle intermediates and toxic metabolites [14]. Secondary active transporters are of two types; symporters, which transport the substrate and the ions in the same direction, and the antiporters, which transport ions and the substrates in opposite directions $[15,16]$. Based on the sequence homology, secondary active transporter proteins responsible for antimicrobial efflux are classified into 4 major families namely i) the major facilitator super family (MFS), ii) the resistance-nodulation-cell division transporter super family (RND), iii) the small multidrug resistant transporter super family (SMR), and iv) the multiple antimicrobial extrusion protein super family (MATE). MFS, RND and SMR groups of efflux pumps are $\mathrm{H}^{+}$/drug antiporters, while the MATE group of efflux proteins are $\mathrm{Na}^{+}$/ drug antiporters $[17,18]$. Apart from these secondary active efflux proteins, bacteria also harbor ATP-binding cassette $(\mathrm{ABC})$ transporters which require hydrolysis of ATP to drive the extrusion of antimicrobial agents from the bacterial cell [19].

\section{Efflux pumps and the antibiotic resistance}

Efflux pumps are widely distributed in Gram-positive and Gramnegative bacteria. The genome of Escherichia coli has about 39 putative efflux pumps, while the corresponding number in the genome of Staphylococcus aureus is $31[20,21]$, although the number of efflux pumps need not necessarily determine the extent of antibiotic resistance [22]. The RND family of efflux pumps is specific to Gramnegative bacteria with AcrAB of Escherichia coli being one of the earliest and well characterized RND efflux pumps. Similarly, MFS efflux pumps are prominent in Gram-positive bacteria. NorA of Staphylococcus aureus is an extensively studied MFS efflux pump [23]. With the realization of the significance of efflux pumps in the resistance of bacteria to antimicrobial compounds and the availability of whole genome sequences of bacteria, several new efflux pumps have been identified and characterized, some of which are clinically very important $[24,25]$. Due to inherent problems in the crystallization of membrane proteins for X-ray crystallography, researchers have largely relied on in silico prediction of the efflux pump structures to understand their structure function relationships [26]. In the context of pathogenic bacteria rapidly gaining antimicrobial resistance, inhibition of efflux activity of MDR efflux pumps is an attractive approach towards restoring the efficacy of antibiotics [27]. Natural compounds such as the plant extracts are preferred owing to their generally non-toxic nature.

\section{Plant-based compounds as potential efflux pump inhibitors}

Before the discovery of antibiotics, treatment of microbial infections relied extensively on herbal medicine, especially in the Asian subcontinent. Plants produce potent antimicrobials as self-defense to overcome bacterial and fungal infections and these substances may possess bactericidal or bacteriostatic properties. Plant-derived compounds are promising as efflux pump inhibitors (EPIs) which can potentially restore the efficacy of antibiotics by blocking the activities of efflux pumps and thus allowing the antibiotics to reach the minimum bactericidal concentration inside the bacterial cell [28]. Since numerous plant-based preparations are known to be antibacterial, analysis of such preparations using modern analytical tools such as the High Performance Liquid Chromatography (HPLC) and Tandem Mass Spectrophotometry (MS/MS) will help to identify the compounds responsible for antimicrobial activities. Several recent reviews have elaborated on the useful ness of plant-derived compounds as potent efflux pump inhibitors [29,30]. EPIs can inhibit efflux pumps by any of the mechanisms depicted in Figure 1. In vitro studies have shown that EPIs can restore the efficacy of an antibiotic by rendering a resistant bacteria susceptible to the antibiotic, reduce the Minimum Inhibitory Concentrations (MICs) of antibiotics and reduce the chances of resistance development when used in combination with the antibiotics [30,31].

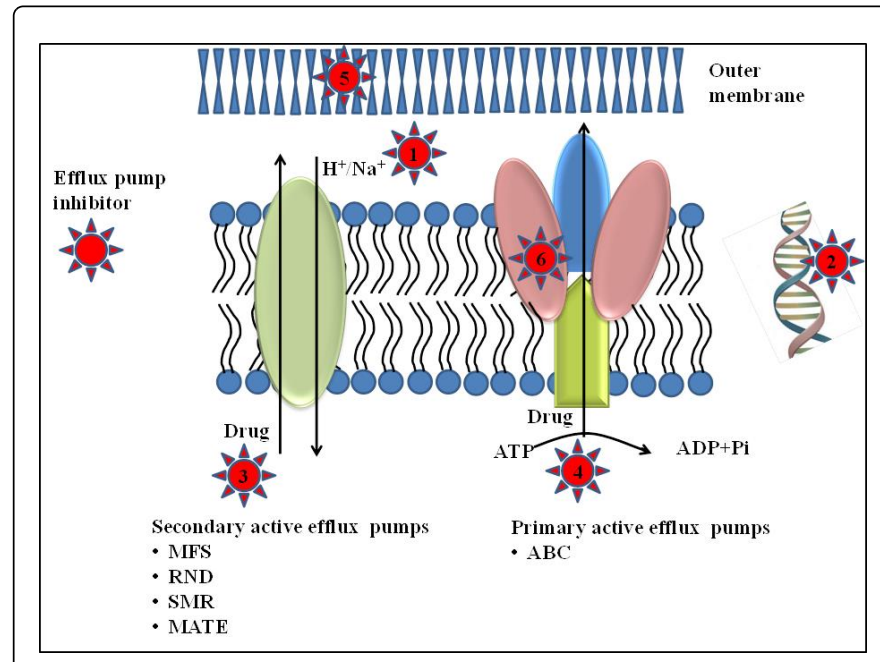

Figure 1: Postulated mechanisms of efflux pump inhibition by EPIs include 1) dissipation of ionic gradient across the cell membrane which is essential for energizing the efflux activity, 2) transcriptional down regulation of the genes encoding efflux pumps, 3) competitive or non-competitive inhibition of substrate binding, 4) disruption of efflux pump energization by interfering with the ATP hydrolysis, 5) altering outer membrane permeability (only in Gram-negative bacteria since Gram-positive bacteria lack an outer membrane), 6) conformational changes in efflux proteins or interference in the assembly of multicomponent efflux pumps (e.g. RND or ABC types) $[32,33]$.

One of the earliest EPI discovered from plant sources is the plant alkaloid reserpine which was shown to be an effective inhibitor of Bmr efflux pump of Bacillus subtilis [34]. However, due to the toxicity of reserpine to humans, its application is restricted to in vitro studies involving efflux pump inhibitions. Belofsky et al. [35] found that a flavonoid and phenolic compounds extracted from the plant Dalea 
versicolor potentiated the activities of berberine and some antibiotics possibly due to efflux pump inhibition. Similarly, isoflavones extracted from Lupinus argenteus was found to potentiate the antibacterial activities of alpha-linolenic acid found in the same plant, as well as the plant antimicrobial berberine and the antibiotic fluoroquinolone (Table 1) [36].

Baicalein derived from the Chinese medicinal plant Scutellaria baicalensis Georgi is known to significantly enhance the activities of $\beta$ lactam antibiotics, tetracycline and tetracycline against MRSA [37]. Diterpenes such as ferruginol extracted from the cones of Chamaecyparis lawsoniana exhibited efflux modulatory activities in methicillin-resistant Staphylococcus aureus and also potentiated oxacillin activity against MRSA [38]. Ferruginol could result in $40 \%$ reduction in ethidium bromide efflux by a MDR strain of Staphylococcus aureus harboring NorA efflux pump [38]. Another study evaluated 13 different phytoalkaloids as efflux inhibitors and found that alkaloids such as quinine, piperine and harmaline exhibited significant efflux inhibitory activities against methicillin resistant Staphylococcus aureus [39]. Similarly, plant alkaloids ellagic and tannic acids not only potentiated the antibacterial activities of novobiocin, coumermycin, chlorobiocin, rifampicin and fusidic acid against Acinetobacter baumannii, but also resulted in the inhibition of efflux activities [40]. Indirubin, extracted from the leaves of Wrightia tinctoria exhibited antibacterial activity against MDR Staphylococcus aureus and $S$. epidermidis, and also inhibited efflux activity similar to reserpine. Although not experimentally determined, the ciproflioxacin potentiation activity of indirubin suggested possible inhibition of NorA efflux pump [41]. Cucurbitane-type triterpenoids extracted from aerial parts of Momordica balsamina significantly inhibited efflux of EtBr by a MRSA strain and Enterococcus faecalis [42]. Coumarins derived from Mesua ferrea have also been found to be potentiators of norfloxacin against clinical strains of Staphylococcus aureus as well as MRSA [43]. These compounds also significantly inhibited efflux activity in a NorA overexpressing strain of Staphylococcus aureus [43]. In a study by Dwivedi et al. [44] 4-hydroxy-a-tetralone (1) isolated from Ammannia spp. along with its semi-synthetic derivatives (1a-1e) was found to reduce the MIC of tetracycline to multidrug resistant Escherichia coli. Using Rhodamine $6 \mathrm{G}$ accumulation assay, it has been shown that the ethanolic leaf extracts of two plant species Callistemon citrinus and Vernonia adoensis from Zimbabwe were inhibitors of efflux pumps of Staphylococcus aureus [45]. In continuation of this finding, it was shown that alkaloids from Callistemon citrinus were more effective in inhibiting the efflux activities in Staphylococcus aureus and Pseudomonas aeruginosa. The mechanism of efflux inhibition involved blocking of ATP-dependent transport of solutes across the membrane [46]. In addition, these extracts increased the permeability of cell membranes of Staphylococcus aureus and Pseudomonas aeruginosa which presumably enhanced the movement of antibiotic across the membrane [45]. Of the several extracts from the roots of the African medicinal plant Zanthoxylum capense Thunb. (Rutaceae), the phenylpropanoid (+)-ailanthoidiol (6) and its ester derivatives exhibited efflux pump inhibitory activities in Staphylococcus aureus and enhance the ciprofloxacin activity against the MRSA strain [47]. Aromatic plant-derived monoterpenic phenols thymol and carvacrol have been reported to be efflux pump inhibitors in food-borne pathogens such as Salmonella enteritidis. Thymol was found to be more effective in reducing the MICs of tetracycline and benzalkonium chloride [48]. Extracts of Anadenanthera colubrina var. cebil enhanced the antimicrobial activities of aminoglycosides against MDR Staphylococcus aureus [49]. Essential oils (EOs) from Salvia fruticosa could reduce the MICs of tetracycline by inhibition of efflux pumps and decrease the expression of tet $(K)$ gene in tetracycline resistant clinical isolates of Salmonella enteritidis [50]. A gallotannin 1,2,6-tri-O-galloyl- $\beta$-D-glucopyranose extracted from Terminalia chebula inhibited efflux pump activity-dependent ethidium bromide extrusion in a multidrug resistant Escherichia coli [51]. A recent reported the LmrS efflux pump inhibitory activity of the spice cumin in a dose dependent manner [52]. Conessine, a steroidal alkaloid compound derived from the plant Holarrhena antidysenterica, exhibited synergestic activities with cefotaxime, levofloxacin, and tetracycline resulting in their significantly reduced MICs to Pseudomonas aeruginosa by inhibiting MexAB-OprM efflux pump [54].

The real time gene expression suggested significant down regulation of ABC-type efflux pump gene yojI and the affinity of the compounds 1 and 1e with YojI was confirmed by molecular docking studies [44]. While the inhibition of efflux activity and potentiation of antibiotics could be ascertained by MIC and ethidium bromide efflux assays, it is relatively difficult to determine if one or more efflux pumps are inhibited, and to identify the target efflux pump. The real time PCR would help to determine if known efflux pumps are under expressed due to the activities of inhibitory compounds. For example, Aghayan et al. [54] investigated the effect of two EPIs Berberine and Palmatine on the expression patterns of mexA, mexB, mexC, mexD, mexE, mexF and mexX in Pseudomonas aeruginosa isolated from burn infections. This study revealed that the effect of these EPIs was more on the MexAB-OprM operon [53].

\begin{tabular}{|l|l|l|l|l|}
\hline Plant source & Active compound & Target bacterium & Target efflux pump & Reference \\
\hline Ammannia spp & $\begin{array}{l}\text { 4-hydroxy-a-tetralone (1) \& semisynthetic } \\
\text { derivatives }\end{array}$ & Escherichia coli & Yojl & {$[44]$} \\
\hline Anadenanthera colubrina & ND & Staphylococcus aureus & ND & {$[49]$} \\
\hline Berberis aetnensis & Pheophorbide & Staphylococcus aureus & Multiple efflux pumps & {$[54]$} \\
\hline Berberis vulgaris & Berberine and Palmatine & Pseudomonas aeruginosa & MexAB-OprM & {$[55]$} \\
\hline $\begin{array}{l}\text { Callistemon citrinus and } \\
\text { Vernonia adoensis }\end{array}$ & Ethanolic leaf extracts & Staphylococcus aureus & $\begin{array}{l}\text { ATP-dependent } \\
\text { pump }\end{array}$ & efflux \\
\hline Capsicum spp. & Capsaicin (8-methyl-N-vanillyl-6-nonenamide) & Staphylococcus aureus & NorA & {$[56]$} \\
\hline Chamaecyparis lawsoniana & Diterpenes (Ferruginol) & Staphylococcus aureus & NorA & {$[38]$} \\
\hline
\end{tabular}


Citation: $\quad$ Rao M, Padyana S, Dipin KM, Kumar S, Nayak BB, et al. (2018) Antimicrobial Compounds of Plant Origin as Efflux Pump Inhibitors: New Avenues for Controlling Multidrug Resistant Pathogens. J Antimicrob Agents 4: 1000159. doi:10.4172/2472-1212.1000159

Page 4 of 6

\begin{tabular}{|c|c|c|c|c|}
\hline Chamaecyparis nootkatensis & phenolic diterpene totarol & Staphylococcus aureus & NorA & {$[57]$} \\
\hline Dalea versicolor & Flavanoid, phenolic compounds & $\begin{array}{l}\text { Staphylococcus aureus, } \\
\text { Bacillus cereus }\end{array}$ & NorA & [36] \\
\hline Holarrhena antidysenterica & Conessine & Pseudomonas aeruginosa & MexAB-OprM & [53] \\
\hline Hypericum olympicum & Compound 1 & Staphylococcus aureus & NorA & {$[58]$} \\
\hline Lupinus argenteus & Isolflavones & Staphylococcus aureus & NorA & [36] \\
\hline Mesua ferrea & Coumarins & Staphylococcus aureus & NorA & [43] \\
\hline Momordica balsamina & Cucurbitane-type triterpenoids & $\begin{array}{l}\text { Staphylococcus aureus, } \\
\text { Enterococcus faecalis }\end{array}$ & - & [42] \\
\hline Persea lingue & Kaempferol rhamnoside & Staphylococcus aureus & NorA & [59] \\
\hline Rosmarinus officinalis) & Abietane diterpenes & Staphylococcus epidermis & $\operatorname{Msr}(\mathrm{A}), \operatorname{Tet}(\mathrm{K})$ & [60] \\
\hline Salvia fruticosa & Essential oils & Staphylococcus aureus & TetK & [50] \\
\hline Scutellaria baicalensis Georgi & Baicalein & Salmonella enteritidis & NorA & [37] \\
\hline Terminalia chebula & $\begin{array}{l}\text { Gallotannin } \\
\text { glucopyranose }\end{array}$ & Escherichia coli & ND & [51] \\
\hline Various aromatic plants & Thymol and carvacrol & Food-borne pathogens & ND & [48] \\
\hline Wrightia tinctoria & Indirubin & $\begin{array}{l}\text { Staphylococcus aureus, } \\
\text { Staphylococcus. } \\
\text { epidermidis }\end{array}$ & NorA & [41] \\
\hline Zanthoxylum capense Thunb. & Phenylpropanoid (+)-ailanthoidiol (6) & Staphylococcus aureus & ND & [47] \\
\hline
\end{tabular}

Table 1: Efflux pump inhibitor compounds from plant sources, their target efflux proteins and the bacterial pathogens inhibited.

The gene expression analysis approach is confounded by the fact that the effect of many efflux inhibitors could be at the protein level or they may be simply acting by diffusing the ionic gradient required for driving the ant port activity [61]. In addition, a bacterium may possess many efflux pumps including several uncharacterized or even unidentified efflux pumps. Microarray is an alternative approach which can help to identify unknown efflux pump genes modulated by the activities of inhibitory compounds. Further, molecular docking is a powerful tool to understand the interactions of natural compounds with the efflux pumps and to identify the actual target sites of inhibitory compounds [62]. In a recent study, 328 secondary plant metabolites were screened for their inhibitory activity against cytoplasmic exporter protein MexB of MexAB-OprM efflux pump of Pseudomonas aeruginosa. In vitro studies showed that $\mathrm{p}$-coumaric acid was able to potentiate the antibacterial activity of ciprofloxacin in MexAB-OprM overexpressing strain of Pseudomonas aeruginosa. Further, in silico studies showed that a p-coumaric acid derivative 4(4-((Z)-2-carboxy-2-((Z)-2,3-dihydrobenzo[e][1,4]diazepin-1-yl)-1(4-hydroxyphenyl)vinylamino) phenylsulfonamido)-2-hydroxybenzoic acid was able to dock more efficiently than the parent compound and could act as a more potent inhibitor of RNA efflux pumps [63].

\section{Conclusions}

The potential role of EPIs in overcoming efflux mediated antibiotic resistance in pathogenic bacteria is increasingly being recognized. EPIs can restore the activities of antibiotics wherever efflux is the sole mechanism of antibiotic resistance. Medicinal plants with known antimicrobial activities can make good candidates to search for potential EPIs. High throughput screening methods have enabled testing of large number of compounds, both natural as well as their semisynthetic or synthetic derivatives. EPIs identified by in vitro approach can be subjected to structural elucidation followed by in silico docking analysis to identify the molecular interactions between the EPIs and their targets. Understanding the molecular interactions will help in modifying EPIs for better binding with their target efflux pumps and hence, more efficient inhibition of efflux pumps. Thus, EPIs are highly promising as potential adjuvants to maintain the efficacy of antibiotics in the treatment of infectious diseases.

\section{References}

1. Lekshmi M, Ammini P, Kumar S, Varela MF (2017) The food production environment and the development of antimicrobial resistance in human pathogens of animal origin. Microorganisms 5: E11.

2. Boeckel TPV, Brower C, Gilbert M, Grenfell BT, Levin SA, et al. (2015) Global trends in antimicrobial use in food animals. Proc Natl Acad Sci USA 112: 5649-5654.

3. Levy SB (1998) The challenge of antibiotic resistance. Sci Am 278: 46-53.

4. Okeke IN, Laxminarayan R, Bhutta ZA, Duse AG, Jenkins P, et al. (2005) Antimicrobial resistance in developing countries. Part I: recent trends and current status. Lancet Infect Dis 5: 481-493.

5. Walsh TR, Toleman MA (2011) The new medical challenge: Why NDM-1? Why Indian? Expert Rev Anti Infect Ther 9: 137-141.

6. Kumar S, Varela MF (2013) Molecular mechanisms of bacterial resistance to antimicrobial agents. In: Microbial pathogens and strategies for combating them: Science, technology and education. A. Méndez-Vilas (Ed.) 2013: 522-534. 
7. Davies J, Davies D (2010) Origins and evolution of antibiotic resistance. Microbiol Mol Biol Rev 74: 417-433.

8. Kumar S, Varela MF (2012) Biochemistry of bacterial multidrug efflux pumps. Int J Mol Sci 13: 4484-4495.

9. Summers AO (2006) Genetic linkage and horizontal gene transfer, the roots of the antibiotic multi-resistance problem. Anim Biotechnol 17: 125-135.

10. George AM, Levy SB (1983). Amplifiable resistance to tetracycline, chloramphenicol and other antibiotics in Escherichia coli: Involvement of a non-plasmid-determined efflux of tetracycline. J Bacteriol 155: 531-540.

11. Alekshun MN, Levy SB (1997) Regulation of chromosomally mediated multiple antibiotic resistance: The mar regulon. Antimicrob Agents Chemother 41: 2067-2075.

12. Webber MA, Buckley M, Randall LP, Woodward MJ, Piddock LJ (2006) Overexpression of marA, soxS and acrB in veterinary isolates of Salmonella enterica rarely correlates with cyclohexane tolerance. J Antimicrob Chemother 57: 673-679.

13. Forrest LR, Krämer R, Ziegler C (2011) The structural basis of secondary active transport mechanisms. Biochim Biophys Acta 1807: 167-188.

14. Boudker O, Verdon G (2010) Structural perspectives on secondary active transporters. Trends Pharmacol Sci 31: 418-426.

15. Marger MD, Saier MH (1993) A major superfamily of transmembrane facilitators that catalyse uniport, symport and antiport. Trends Biochem Sci 18: 13-20.

16. Nelson N (1994) Energizing porters by proton-motive force. Exp Biol 196: 7-13.

17. Paulsen IT, Brown MH, Skurray RA (1996) Proton-dependent multidrug efflux systems. Microbiol Rev 60: 575-608.

18. Murakami S, Yamaguchi A (2003) Multidrug-exporting secondary transporters. Curr Opin Struct Biol 13: 443-452.

19. Davidson AL, Maloney PC (2007) ABC transporters: How smal machines do a big job. Trends Microbiol 15: 448-455.

20. Paulsen IT, Sliwinski MK, Saier MH (1998) Microbial genome analyses: Global comparisons of transport capabilities based on phylogenies, bioenergetics and substrate specificities. J Mol Biol 277: 573-592.

21. Schindler BD, Manso EF, DeMarco CE, Kosmidis C, Matta V, et al. (2015) Analyses of multidrug efflux pump-like proteins encoded on the Staphylococcus aureus chromosome. Antimicrob Agents Chemother 59: 747-748.

22. Youenou B, Bonté SF, Bodilis J, Brothier E, Dubost A, et al. (2015) Comparative genomics of environmental and clinical Stenotrophomonas maltophilia strains with different antibiotic resistance profiles. Genome Biol Evol 7: 2484-2505.

23. Blanco P, Amado SH, Calderon JAR, Corona F, Lira F, et al. (2016) Bacterial multidrug efflux pumps: Much more than antibiotic resistance determinants. Microorganisms 4: E14.

24. Andersen JL, He GX, Kakarla P, Ranjana KC, Kumar S, et al. (2015) Multidrug efflux pumps from Enterobacteriaceae, Vibrio cholerae and Staphylococcus aureus bacterial food pathogens. Int J Environ Res Public Health 12: 1487-1547.

25. Schindler BD, Kaatz GW (2016) Multidrug efflux pumps of Grampositive bacteria. Drug Resist Updat 27: 1-13

26. Sharma S, Kumar M, Sharma S, Nargotra A, Koul S, et al. (2010) Piperine as an inhibitor of Rv1258c, a putative multidrug efflux pump of Mycobacterium tuberculosis. J Antimicrob Chemother 65: 1694-1701.

27. Kumar S, He G, Kakarla P, Shrestha U, Ranjana KC, et al. (2016) Bacterial multidrug efflux pumps of the major facilitator superfamily as targets for modulation. Infect Disord Drug Targets 16: 28-43.

28. Marquez B (2005) Bacterial efflux systems and efflux pumps inhibitors. Biochimie 87: 1137-1147.

29. Prasch S, Bucar F (2015) Plant derived inhibitors of bacterial efflux pumps: An update. Phytochem Rev 14: 961-974.

30. Mahmood HY, Jamshidi S, Sutton JM, Rahman KM (2016) Current advances in developing inhibitors of bacterial multidrug efflux pumps. Curr Med Chem 23: 1062-1081.
31. Kaatz GW (2005) Bacterial efflux pump inhibition: a potential means to recover clinically relevant activity of substrate antimicrobial agents. Curr Opin Investig Drugs 6: 191-198.

32. Pagès JM, Amaral L (2009) Mechanisms of drug efflux and strategies to combat them: challenging the efflux pump of Gram-negative bacteria. Biochim Biophys Acta 1794: 826-833.

33. Marino LB, Miyata M, Souza PC, Leite CQF, Pavan FR (2014) Drug discovery for TB: Frontiers and perspectives. Front Anti-Infect Drug Discov Edn 1 29: 3-31.

34. Ahmed M, Borsch CM, Neyfakh AA, Schuldiner S (1993) Mutants of the Bacillus subtilis multidrug transporter Bmr with altered sensitivity to the antihypertensive alkaloid reserpine. J Biol Chem 268: 11086-11089.

35. Belofsky G, Percivill D, Lewis K, Tegos GP, Ekart J (2004) Phenolic metabolites of Dalea versicolor that enhance antibiotic activity against model pathogenic bacteria. J Nat Prod 67: 481-484.

36. Morel C, Stermitz FR, Tegos G, Lewis K (2003) Isoflavones as potentiators of antibacterial activity. J Agric Food Chem 51: 5677-5679.

37. Chan BC, Ip M, Lau CB, Lui SL, Jolivalt C, et al. (2011) Synergistic effects of baicalein with ciprofloxacin against NorA over-expressed methicillinresistant Staphylococcus aureus (MRSA) and inhibition of MRSA pyruvate kinase. J Ethnopharmacol 137: 767-773.

38. Smith EC, Williamson EM, Wareham N, Kaatz GW, Gibbons S (2007) Antibacterials and modulators of bacterial resistance from the immature cones of Chamaecyparis lawsoniana. Phytochemistry 68: 210-217.

39. Mohtar M, Johari SA, Li AR, Isa MM, Mustafa S, et al. (2009) Inhibitory and resistance-modifying potential of plant-based alkaloids against methicillin-resistant Staphylococcus aureus (MRSA). Curr Microbiol 59: 181-186.

40. Chusri S, Villanueva I, Voravuthikunchai SP, Davies J (2009) Enhancing antibiotic activity: A strategy to control Acinetobacter infections. J Antimicrob Chemother 64: 1203-1211.

41. Ponnusamy K, Ramasamy M, Savarimuthu I, Paulraj MG (2010) Indirubin potentiates ciprofloxacin activity in the NorA efflux pump of Staphylococcus aureus. Scand J Infect Dis 42: 500-505.

42. Ramalhete C, Spengler G, Martins A, Martins M, Viveiros M, et al. (2011) Inhibition of efflux pumps in methicillin-resistant Staphylococcus aureus and Enterococcus faecalis resistant strains by triterpenoids from Momordica balsamina. Int J Antimicrob Agents 37: 70-74.

43. Roy SK, Kumari N, Pahwa S, Agrahari UC, Bhutani KK, et al. (2013) NorA efflux pump inhibitory activity of coumarins from Mesua ferrea. Fitoterapia 90: 140-150.

44. Dwivedi GR, Upadhyay HC, Yadav DK, Singh V, Srivastava SK, et al. (2014) 4-Hydroxy- $\alpha$-tetralone and its derivative as drug resistance reversal agents in multi-drug resistant Escherichia coli. Chem Biol Drug Des 3: 482-492.

45. Chitemerere TA, Mukanganyama S (2014) Evaluation of cell membrane integrity as a potential antimicrobial target for plant products. BMC Complement Altern Med 14: 278.

46. Mabhiza D, Chitemerere T, Mukanganyama S (2016) Antibacterial properties of alkaloid extracts from Callistemon citrinus and Vernonia adoensis against Staphylococcus aureus and Pseudomonas aeruginosa. Int J Med Chem 2016: 1-7.

47. Cabral V, Luo X, Junqueira E, Costa SS, Mulhovo S, et al. (2015) Enhancing activity of antibiotics against Staphylococcus aureus: Zanthoxylum capense constituents and derivatives. Phytomedicine 22: 469-476.

48. Miladi H, Zmantar T, Chaabouni Y, Fedhila K, Bakhrouf A, et al. (2016) Antibacterial and efflux pump inhibitors of thymol and carvacrol against food-borne pathogens. Microb Pathog 99: 95-100.

49. Barreto HM, Coelho KM, Ferreira JH, Dos Santos BH, Abreu AP, et al. (2016) Enhancement of the antibiotic activity of aminoglycosides by extracts from Anadenanthera colubrine (Vell.) Brenan var. cebil against multi-drug resistant bacteria. Nat Prod Res 30: 1289-1292.

50. Chovanová R, Mezovská J, Vavebrková Š, Mikulášová M (2015) The inhibition the Tet(K) efflux pump of tetracycline resistant Staphylococcus 
Citation: $\quad$ Rao M, Padyana S, Dipin KM, Kumar S, Nayak BB, et al. (2018) Antimicrobial Compounds of Plant Origin as Efflux Pump Inhibitors: New Avenues for Controlling Multidrug Resistant Pathogens. J Antimicrob Agents 4: 1000159. doi:10.4172/2472-1212.1000159

Page 6 of 6

epidermidis by essential oils from three Salvia species. Lett Appl Microbiol 61: 58-62.

51. Bag A, Chattopadhyay RR (2014) Efflux-pump inhibitory activity of a gallotannin from Terminalia chebula fruit against multidrug-resistant uropathogenic Escherichia coli. Nat Prod Res 28: 1280-1283.

52. Kakarla P, Floyd J, Mukherjee M, Devireddy AR, Inupakutika MA, et al (2017) Inhibition of the multidrug efflux pump LmrS from Staphylococcus aureus by cumin spice Cuminum cyminum. Arch Microbiol 199: 465-474

53. Siriyong T, Srimanote P, Chusri S, Yingyongnarongkul BE, Suaisom C, et al. (2017) Conessine as a novel inhibitor of multidrug efflux pump systems in Pseudomonas aeruginosa. BMC Complement Altern Med 17: 405.

54. Musumeci R, Speciale A, Costanzo R, Annino A, Ragusa S, et al. (2003) Berberis aetnensis C. Presl. extracts: Antimicrobial properties and interaction with ciprofloxacin. Int J Antimicrob Agents 22: 48-53.

55. Aghayan SS, Mogadam KH, Fazli M, Sarokhalil DD, Khoramrooz SS, et al. (2017) The Effects of Berberine and Palmatine on efflux pumps inhibition with different gene patterns in Pseudomonas aeruginosa isolated from burn infections. Avicenna J Med Biotechnol 9: 2-7.

56. Kalia NP, Mahajan P, Mehra R, Nargotra A, Sharma JP, et al. (2012) Capsaicin, a novel inhibitor of the NorA efflux pump, reduces the intracellular invasion of Staphylococcus aureus. J Antimicrob Chemother 67: 2401-2408.
57. Smith EC, Kaatz GW, Seo SM, Wareham N, Williamson EM, et al. (2007) The phenolic diterpene totarol inhibits multidrug efflux pump activity in Staphylococcus aureus. Antimicrob Agents Chemother 51: 4480-4483.

58. Shiu WK, Malkinson JP, Rahman MM, Curry J, Stapleton P, et al. (2013) A new plant-derived antibacterial is an inhibitor of efflux pumps in Staphylococcus aureus. Int J Antimicrob Agents 42: 513-518.

59. Holler JG, Christensen SB, Slotved HC, Rasmussen HB, Gúzman A, et al. (2012) Novel inhibitory activity of the Staphylococcus aureus NorA efflux pump by a kaempferol rhamnoside isolated from Persea lingue Nees. J Antimicrob Chemother 67: 1138-1144.

60. Gibbons S, Oluwatuyi M, Kaatz GW (2003) A novel inhibitor of multidrug efflux pumps in Staphylococcus aureus. J Antimicrob Chemother 51: 13-17.

61. Sana AMO, Repetto V, Moreno S (2013) Carnosic acid is an efflux pumps modulator by dissipation of the membrane potential in Enterococcus faecalis and Staphylococcus aureus. World J Microbiol Biotechnol 29: 137-144.

62. Ramaswamy VK, Cacciotto P, Malloci G, Vargiu AV, Ruggerone P (2017) Computational modelling of efflux pumps and their inhibitors. Essays Biochem 61: 141-156.

63. Choudhury D, Talukdar AD, Chetia P, Bhattacharjee A, Choudhury MD (2016) Screening of natural products and derivatives for the identification of RND efflux pump inhibitors. Comb Chem High Throughput Screen 19: 705-713. 